\section{Mobile-phone Movies to Help Capture Physical Manifestations}

\author{
Kazuhiko Kotani ${ }^{1}$, Yasuhiko Asai ${ }^{2}$ and Youichi Kurozawa ${ }^{1}$
}

Key words: mobile technologies, sign and symptom, epilepsy, convulsion, home care

\section{(DOI: 10.2169/internalmedicine.46.0198)}

To the Editor Recently, a new application of mobile technologies, such as the mobile phone and mobile camera, to the medical field (e.g., health/medical care and welfare services) has been considered (1-4). However, because of the new trend, their practicality and usefulness in the medical field have yet to be well established.

Recently, we encountered a patient who has epilepsy-like fits in which the usage of a mobile phone movie was applicable. The patient was a 16-year-old boy with a history of cerebral bleeding since birth. He had taken an anticonvulsant for epileptic fits as sequelae following the cerebrovascular disease. Because the patient did not have any epileptic episodes for an extended period of time, tapering down the dosage of the anti-convulsant was tried. During the time of reduced dosage, an instant stop of eye movement or a faint involuntary movement of the right arm (possibly epilepsy-like fits lasting about 1-2 minutes), was rarely observed at home. His mother orally complained about his physical manifestations in the outpatient room, but the physician was not fully able to understand it. The mother also refrained from many accusations to maintain a good patientdoctor relationship. During an episode, the mother captured his epilepsy-like fit at home using a mobile-phone movie. When the movie reproduced his episodic manifestation in the outpatient room, the physician was able to understand and accept his conditions, and stopped the tapering and arranged a return to the regular dosage of anti-convulsant. The epilepsy-like fit types seemed difficult to diagnose simply by the captured manifestations. Through further examinations, non-epileptic disorders such as neurosis, metabolic diseases and migraine were ruled out, and electroencephalogram findings indicating spikes of the temporal/occipital lobe were detected during an intermittent period of fit, speculating at a symptomatic complex partial seizure (although the monitoring of electroencephalograms with videos is necessary for a more definitive diagnosis).

In this paper, we reported a patient with epilepsy-like episodes, who had the first chance to help diagnose the patient using clinical signs captured by mobile phone movies outside of the medical environment. This may suggest the practicality and usefulness of mobile phone movies to apply to the medical field. His neurological manifestations were faint, instant and not at-the-fixed-moment, so unless they actually occured before the physician's very eyes, these manifestations might be overlooked. In such cases the usage of mobile phone movies could especially help medical decisions. Mobile phones with movie capability have become common nowadays; accordingly an application of mobile phone movies to the medical field might prove to be useful for various cases.

This method appears to have some disadvantages: e.g. slight changes in manifestations are not easy to capture. The evaluation of small details is becoming more possible with the progress in color and high-quality displays on mobile phones, while the quantitative evaluation will be a future challenge. To capture clinical manifestations by nonprofessionals at home may be improper for medical decision making due to the lack of medical focus. Since the assessment can not be confirmed, some physicians may be resistant to accept the findings by mobile phone movies within their medical decision systems, leading to spoil the patientdoctor relationship. Security and privacy issues also remain. In contrast, there appears to be some advantages: e.g. mobility, instance, convenience, easy operation, visualization (often repeated confirmation), data sending and for record keeping (4). Anyway, based on our experience, there would be some cases in which to capture physical manifestations by mobile phone movies provides, at least, a first step to disease management. We can not ignore the role of mobile technologies in relation to the medical field.

\title{
References
}

1. Maglaveras N, Koutkias V, Chouvarda I, et al. Home care delivery through the mobile telecommunications platform: the Citizen Health System (CHS) perspective. Int J Med Inform 68: 99-111, 2002.
2. Collins RL, Kashdan TB, Gollnisch G. The feasibility of using cellular phones to collect ecological momentary assessment data: application to alcohol consumption. Exp Clin Psychopharmacol 11: 73-78, 2003.

\footnotetext{
${ }^{1}$ Division of Health Administration and Promotion, Faculty of Medicine, Tottori University, Yonago and ${ }^{2}$ Division of Dermatology, Tsuruga City Hospital, Tsuruga

Received for publication March 28, 2007; Accepted for publication May 2, 2007

Correspondence to Dr. Kazuhiko Kotani, kakotani@grape.med.tottori-u.ac.jp
} 
DOI: $10.2169 /$ internalmedicine.46.0198

3. Wang DH, Kogashiwa M, Ohta S, Kira S. Validity and reliability of a dietary assessment method: the application of a digital camera with a mobile phone card attachment. J Nutr Sci Vitaminol 48: 498-504, 2002.
4. Kotani K, Morii M, Asai Y, Sakane N. Application of mobilephone cameras to home health care and welfare in the elderly: experience in a rural practice. Aust J Rural Health 13: 193-194, 2005.

(C) 2007 The Japanese Society of Internal Medicine http://www.naika.or.jp/imindex.html 